\title{
Erratum: Pagonabarraga, J.; et al. A Spanish Consensus on the Use of Safinamide for Parkinson's Disease in Clinical Practice. Brain Sci. 2020, 10, 176
}

\author{
Javier Pagonabarraga ${ }^{1,2,3, *}$, José Matías Arbelo ${ }^{4,5}$, Francisco Grandas ${ }^{6,7}$, \\ Maria-Rosario Luquin 8,9 , Pablo Martínez Martín 10,11, Maria Cruz Rodriguez-Oroz ${ }^{12,13}$, \\ Francesc Valldeoriola ${ }^{14,15}$ and Jaime Kulisevsky 1,2,3,16,17 \\ 1 Movement Disorders Unit, Neurology Department, Hospital de la Santa Creu i Sant Pau, 08041 Barcelona, \\ Spain; jkulisevsky@santpau.cat \\ 2 Department of Medicine, Autonomous University of Barcelona, 08193 Barcelona, Spain \\ 3 Centro de Investigación en Red sobre Enfermedades Neurodegenerativas (CIBERNED), 28031 Madrid, Spain \\ 4 Movement Disorders Unit, Neurology Department, Hospital Universitario San Roque, 35001 Las Palmas, \\ Spain; jmarbelo@gmail.com \\ 5 Department of Medicine, Universidad Fernando Pessoa-Canarias, 35450 Las Palmas, Spain \\ 6 Movement Disorders Unit-CSUR, Neurology Department, Hospital General Universitario Gregorio \\ Marañón, 28007 Madrid, Spain; francisco.grandas@salud.madrid.org \\ 7 Department of Medicine, Universidad Complutense de Madrid, 28040 Madrid, Spain \\ 8 Movement Disorders Unit, Clínica Universidad de Navarra (CUN), 31008 Pamplona, Spain; rluquin@unav.es \\ 9 Navarra Institute for Health Research (IdiSNA), 31008 Pamplona, Spain \\ 10 Instituto de Salud Carlos III, 28029 Madrid, Spain; pmm650@hotmail.com \\ 11 Centro de Investigación Biomédica en Red sobre Enfermedades Neurodegenerativas (CIBERNED), \\ 28031 Madrid, Spain \\ 12 Neurology and Neuroscience Unit, Clínica Universidad de Navarra (CUN), 31008 Pamplona, Spain; \\ mcroroz@unav.es \\ 13 Centre for Applied Medical Research (CIMA), 31008 Pamplona, Spain \\ 14 Neurosciences Institut, Hospital Clinic de Barcelona, 08036 Barcelona, Spain; FVALLDE@clinic.ub.es \\ 15 Department of Medicine, Universitat de Barcelona, 08036 Barcelona, Spain \\ 16 Biomedical Research Institute (IIB-Sant Pau), 08041 Barcelona, Spain \\ 17 Department of Medicine, Universitat Oberta de Catalunya, 08018 Barcelona, Spain \\ * Correspondence: JPagonabarraga@santpau.cat; Tel.: +34-647-618-844; Fax: +34-935-565-748
}

Received: 11 May 2020; Accepted: 15 May 2020; Published: 22 May 2020

We would like to submit the following erratum to our recently published paper [1] due to the errors in the abstract. We request (1) the removal of the word "motor" from the first line of the abstract, so that the sentence reads: "Safinamide is an approved drug for the treatment of fluctuations in Parkinson's disease (PD)", and (2) that the word "OFF" is changed to "ON" in line 6 of the abstract, so that the sentence reads: "Safinamide significantly improves the mean daily ON time without troublesome dyskinesias."

We apologize for any inconvenience caused to our readers.

Conflicts of Interest: The authors declare no conflict of interest. 


\section{Reference}

1. Pagonabarraga, J.; Arbelo, J.M.; Grandas, F.; Luquin, M.-R.; Martínez Martín, P.; Rodríguez-Oroz, M.C.; Valldeoriola, F.; Kulisevsky, J. A Spanish Consensus on the Use of Safinamide for Parkinson's Disease in Clinical Practice. Brain Sci. 2020, 10, 176. [CrossRef] [PubMed] 